University of Nebraska - Lincoln

DigitalCommons@University of Nebraska - Lincoln

\title{
Electronic Structure Evidence for All-Trans Poly(methylvinylidene cyanide)
}

Jie Xiao

University of Nebraska-Lincoln, jie.xiao@helmholtz-berlin.de

Matt Poulsen

University of Nebraska-Lincoln, map@suiter.com

D. Sahadeva Reddy

University of Nebraska-Lincoln

James M. Takacs

University of Nebraska-Lincoln, jtakacs1@unl.edu

Yaroslav B. Losovyj

Louisiana State University at Baton Rouge, ylozovyy@indiana.edu

See next page for additional authors

Follow this and additional works at: https://digitalcommons.unl.edu/physicsdowben

Part of the Physics Commons

Xiao, Jie; Poulsen, Matt; Reddy, D. Sahadeva; Takacs, James M.; Losovyj, Yaroslav B.; and Dowben, Peter A., "Electronic Structure Evidence for All-Trans Poly(methylvinylidene cyanide)" (2008). Peter Dowben Publications. 213.

https://digitalcommons.unl.edu/physicsdowben/213

This Article is brought to you for free and open access by the Research Papers in Physics and Astronomy at DigitalCommons@University of Nebraska - Lincoln. It has been accepted for inclusion in Peter Dowben Publications by an authorized administrator of DigitalCommons@University of Nebraska - Lincoln. 
Authors

Jie Xiao, Matt Poulsen, D. Sahadeva Reddy, James M. Takacs, Yaroslav B. Losovyj, and Peter A. Dowben 
Published in Polymer Engineering and Science 48 (2008), pp. 1649-1654; doi 10.1002/pen

Copyright (C) 2008 Society of Plastics Engineers; published by John Wiley \& Sons, Inc.

Used by permission. http://www.interscience.wiley.com

Research sponsored by National Science Foundation contract grant numbers CHE-0415421 and CHE-0650453.

\title{
Electronic Structure Evidence for All-Trans Poly(methylvinylidene cyanide)
}

\author{
Jie Xiao, ${ }^{1,2}$ Matt Poulsen, ${ }^{1,2}$ D. Sahadeva Reddy, ${ }^{2,3}$ J. M. Takacs, ${ }^{2,3}$ \\ Y. B. Losovyj, ${ }^{4}$ and P. A. Dowben ${ }^{1,2}$ \\ ${ }^{1}$ Department of Physics and Astronomy, Behlen Laboratory of Physics, \\ University of Nebraska-Lincoln, Lincoln, Nebraska 68588-0111 \\ ${ }^{2}$ Nebraska Center for Materials and Nanoscience, University of Nebraska-Lincoln, Lincoln, Nebraska 68588-0111 \\ ${ }^{3}$ Department of Chemistry, University of Nebraska-Lincoln, Lincoln, Nebraska 68588-0304 \\ ${ }^{4}$ Center for Advanced Microstructures and Devices, Jefferson Hwy, Louisiana State University, Baton Rouge, Louisiana 70806 \\ Corresponding author - Jie Xiao, email xiaojie@unlserve.unl.edu
}

\begin{abstract}
On the basis of a comparison of theoretical quantum calculations, by both semiempirical and ab initio methods, with photoemission and inverse photoemission results, we suggest that polymethylvinylidenecyanide (PMVC) adopts an all-trans conformation with few, if any, alternating trans-gauche carbon-carbon bond arrangements. The comparison of theory with the available photoemission and inverse photoemission excludes the presence of a significant fraction of gauche bonds in the polymer chains, indicative of the all-trans conformation, with dipoles all aligned.
\end{abstract}

\section{Introduction}

Photoemission, particularly light polarization dependent photoemission [1, 2], can be very successful in establishing that the highly dipole-ordered polymers, prepared by Langmuir Blodgett techniques [3, 4], have dipoles oriented along the surface normal, particularly at temperature well below room temperature ( $100 \mathrm{~K})$. The high-quality ultrathin crystalline ferroelectric films, like the copolymer poly(vinylidene fluoride with trifluoroethylene) P(VDFTrFE) [3-5], have a dipole ordered ferroelectric phase, the $\beta$ phase (parallel dipoles) [3]. Because of symmetry breaking at the surface, light polarization dependent photoemission does not, however, distinguish from up or down oriented dipoles per se $[2,6]$. Further, the value of light polarization dependent angle resolved photoemission is quite limited if the molecular point group symmetry is very low, even if the organic layer is well ordered [7].

Distinguishing between the $\beta$ phase (parallel dipoles) and the $\alpha$ phase (antiparallel dipoles) in P(VDF-TrFE) thin films is rather straightforward, as the latter is paraelectric with no net polarization. The determination of dipole ordering is, however, complicated at the surface layer, as there exists a surface ferroelectric phase transition from all-trans ( $\beta$ phase) to a phase where period doubling of the surface structure occurs (involving four $-\left(\mathrm{CH}_{2}-\mathrm{CF}_{2}\right)-$ monomers) and ordering at the surface is diminished [in the temperature region of $20^{\circ} \mathrm{C}$ for $\left.\mathrm{P}(\mathrm{VDF}-\mathrm{TrFE}), 70 \%: 30 \%\right]$ $[8,9]$.

For the highly dipole-ordered polymer poly(methylvinylidene cyanide) (PMVC), $\left(-\left(\mathrm{CH}\left(\mathrm{CH}_{3}\right)-\mathrm{C}(\mathrm{CN})_{2}\right)_{n}-\right)$, the type of dipole order at the surface is only weakly established. We found evidence for strong intramolecular band structure [7], but this does not provide a clear indication of the type of dipole ordering along the surface normal: both an all-trans and some type of alternating transgauche conformations are possible from the experimental band mapping data. The band structure is consistent with a real space periodicity of three monomer repeats, i.e. for $\left(-\left(\mathrm{CH}\left(\mathrm{CH}_{3}\right)-\mathrm{C}(\mathrm{CN})_{2}\right)_{n}-\right), n=3$, and this tends to exclude some ordered alternating trans-gauche conformation, independent experimental confirmation of a mostly all-trans configuration would be better. The strong evidence of band structure also excludes a random azimuthal dipole orientation with respect to the polymer chain backbone. The scanning tunneling microscopy (STM) images [7] are also more consistent with an all-trans configuration, but regrettably are also not compelling evidence in this regard. There are difficulties in obtaining X-ray diffraction (XRD) from PMVC thin films [10], while light polarization dependent 

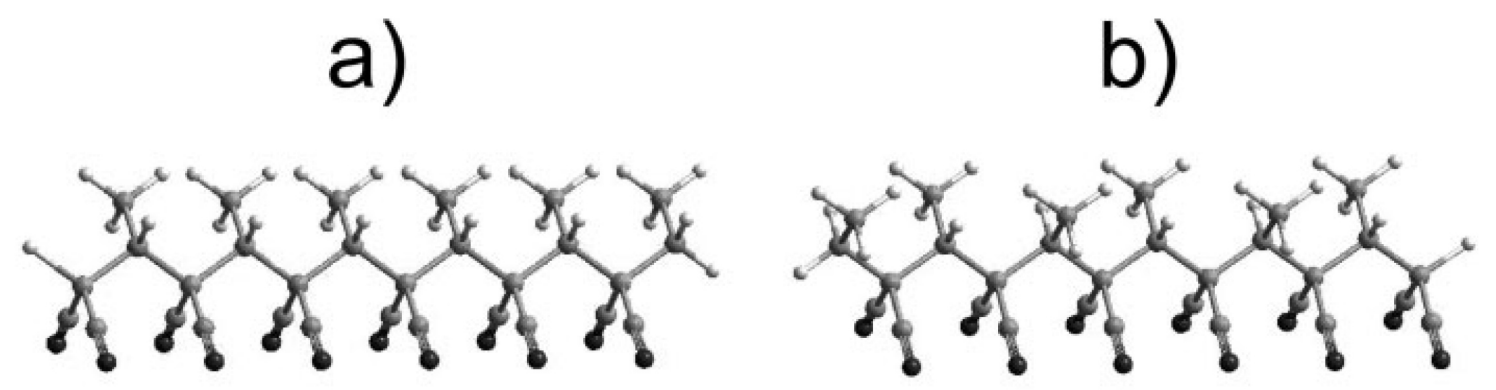

\section{c)}

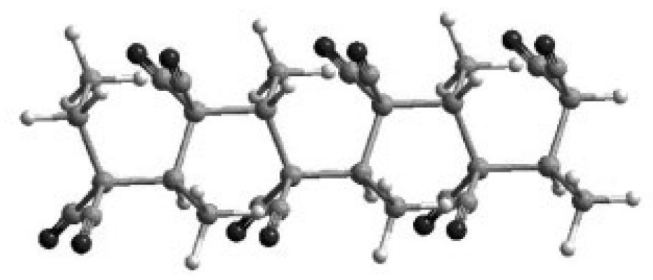

d)

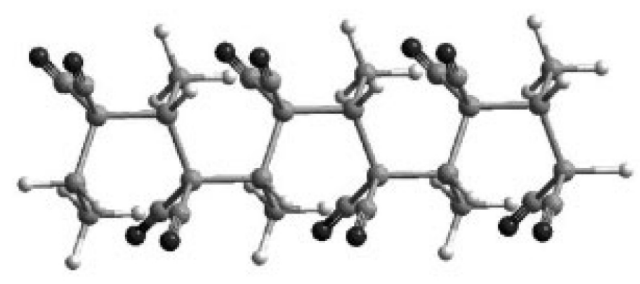

Figure 1. The schematic representations of chain segments of PMVC for some of the possible different configurations for PMVC considered here: (a) all-trans with methyl groups lined up; (b) all-trans with methyl groups on both sides alternatively; (c) alternating transgauche with methyl groups on both sides; and (d) alternating trans-gauche with methyl groups on one side. Nitrogen is shown to be darker than carbon or hydrogen.

photoemission and infrared spectroscopy are inconclusive as to the dipole ordering, because of the very low local point group symmetry [7]. Furthermore, because the local dipoles do not appear as readily to "rotate" for PMVC [10], the issue of dipole ordering remains poorly addressed. The actual dipole ordering will affect the dielectric properties of PMVC thin films and could provide insight as to the origin of the crystalline polymer band structure critical points: i.e. the correspondence to a real space periodicity of three monomer repeats, i.e. $n=3$ for $-\left(\mathrm{CH}\left(\mathrm{CH}_{3}\right)-\mathrm{C}(\mathrm{CN})_{2}\right)_{n}-$.

To judge better the PMVC geometric structure with regard to the dipole ordering, we have proceeded to make a comparison between model calculations of the electronic structure and combined photoemission and inverse photoemission results. Both the semiempirical and ab initio calculations for the all-trans and alternating trans-gauche conformations, for the different positions of methyl groups were performed for likely configurations of PMVC, as summarized in Figure 1. We find that changing the positions of methyl groups -i.e. regio-regular or alternating site positions versus a low symmetry chiral placement (all methyl groups on one side) - does not alter the expected electronic structure, but altering the dipole orientation does make the electronic structure quite different from experiment.

\section{Experimental}

Films of PMVC $\left[-\left(\mathrm{CH}\left(\mathrm{CH}_{3}\right)-\mathrm{C}(\mathrm{CN})_{2}\right)_{n}-\right]$ were prepared using the Langmuir-Blodgett (LB) technique from a water subphase $[3,4,10]$. The PMVC polymer with weight-averaged molecular weight $\left(M_{\mathrm{w}}\right)=2780$ was dissolved at 0.07 wt $\%$ in a 90:10 mixture of chloroform and dimethylsulfoxide. For each material, a monolayer was dispersed on the surface of a NIMA model 622C LB trough filled with ultrapure $(>18 \mathrm{M} \Omega \mathrm{cm})$ water and compressed to a surface pressure of $5 \mathrm{mN} / \mathrm{m}^{2}$. The LB films were deposited on substrates of freshly cleaved pyrolytic graphite wafers by the horizontal (Schaefer) variation of the LB technique, repeating five times to make a film of five nominal monolayers. Film preparation methods are described in greater detail elsewhere [10]. The LB method produces films of PMVC with a high degree of surface crystallinity, as is evident from STM taken at room temperature [7], and experimental band structure mappings [7]. The PMVC thin films prepared in this manner shows at the very least paracrystallinity (the polymer chain are highly aligned), if not crystallinity. Although annealed in vacuum, the films are not annealed to temperature anywhere approaching a melt (annealing temperatures $150^{\circ} \mathrm{C}$ ) and no loss of order was observed.

The ultraviolet photoemission spectra (UPS) and inverse photoemission spectra (IPES) were undertaken to study the molecular orbital placement of both occupied and unoccupied orbitals of the polymers. In both photoemission and inverse photoemission measurements, the binding energies are referenced with respect to the Fermi edge of gold or tantalum, in intimate contact with the sample surface. The IPES were obtained by using variable incident energy elec- 
trons while measuring the emitted photons at a fixed energy $(9.7 \mathrm{eV})$ using a Geiger-Müller detector [7-9]. The instrumental line width is $\sim 400 \mathrm{meV}$, as described elsewhere [7-9]. The light polarization dependent angle-resolved UPS (ARUPS) were carried out using synchrotron light at $55 \mathrm{eV}$ photon energy, dispersed by a $3 \mathrm{~m}$ toroidal grating monochromator, at the Center for Advanced Microstructures and Devices (CAMD) in Baton Rouge, Louisiana [11], employing a hemispherical electron energy analyzer with an angular acceptance of $\pm 1^{\circ}$, as described in detail elsewhere $[12,13]$.

The high resolution photoemission studies were carried out on separate $3 \mathrm{~m}$ normal incidence (NIM) beamline that consists of water cooled ellipsoidal entrance mirror with a $70 \mathrm{mrad}$ acceptance angle of horizontal radiation from a dipole magnet at CAMD [14]. This NIM monochromator is combined with an ARUPS endstation, which consists of a magnetic field shielded UHV chamber with the electron energy analyzer (Scienta SES200 electron energy analyzer) [14]. Both gas phase resolution tests and solid-state sample show that the combined resolution (including beamline and analyzer) is $9 \mathrm{meV}$ or less for the Fermi edge of gold films at the lowest possible temperature we have obtained and better than $5 \mathrm{meV}$ ultimate electron energy analyzer resolution for Ar $3 p$ level using He I radiation [14].

\section{Computational Details}

Both semiempirical and $a b$ initio LCAO methods, both based on Hartree-Fock formalism, were used. The semiempirical method NDO-PM3 (neglect of differential diatomic overlap, parametric model number 3 ), which has proved to be a successful, although simplistic, approach for modeling the electronic structure for organic molecules [15], was undertaken to calculate the electronic structure of PMVC. As its name indicates, two-electron integrals are completely omitted in this method. Nonetheless, semiempirical model calculations still produce a density of states (DOSs) that is in generally good agreement with the experimental combined photoemission and inverse photoemission data [7], as seen in Figure 2.

Because two-electron integrals are excluded in the semiempirical PM3 calculation, a more accurate methodi.e., ab initio LCAO/LDA calculations that contains twoelectron integrals-has also been undertaken to calculate the electronic structure for the four different conformations of PMVC. The ab initio method involves a more fundamental approach to solving the Schrödinger equation than does the semiempirical method, although there are some assumptions implicit in such calculations [16]. The basic approximation of $a b$ initio calculation is that the total wave function is a single Slater determinant and the resultant expression of the molecular orbitals is a linear combination of atomic orbital basis functions (LCAO): in other word, $a b$ initio calculation can be initiated once a basis for the LCAO is chosen. We chose 3-21G basis set which are Gaussian functions and can simplify the two- electron integral calculation. These approaches are, of course, compromises. Another approach would be to use a semiempirical hybrid functional like B3LYP $[17,18]$ which is seen to work well in solid-state calculations for modeling the band gap [19], but density functional theory (DFT) is notorious for underestimating the band gap sometimes by a factor 2 or more [16], particularly for molecular systems, although DFT is excellent for estimating total energies in systems similar to PMVC [20].

A series of molecular orbitals, whose energy levels are with respect to the vacuum energy $E_{\mathrm{vac}}$ were obtained by the calculations of these two methods (LCAO and PM3) on the short segments of PMVC chains. A model DOSs was obtained by applying equal Gaussian envelopes of $1 \mathrm{eV}$ full width half maximum to each calculated molecular orbital energy (eigenvalue) to account for the solid state broadening in photoemission and then summing. These model DOSs calculations are rigidly shifted in energy by $5.3 \mathrm{eV}$ and then compared with the combined photoemission and inverse photoemission data from PMVC thin films, taken at room temperature [7], as indicated in Figure 2. The 5.3 $\mathrm{eV}$ energy shift, which is, for the most part, representative of work function $\Phi$ equal to the difference of vacuum energy $E_{\mathrm{vac}}$ and Fermi level $E_{\mathrm{F}}$, is applied to the calculated electronic structure uniformly. No corrections are made for final state effects or matrix element effects in either calculation, so the comparison with experiment is simplistic, but nonetheless still often successful [21-27].

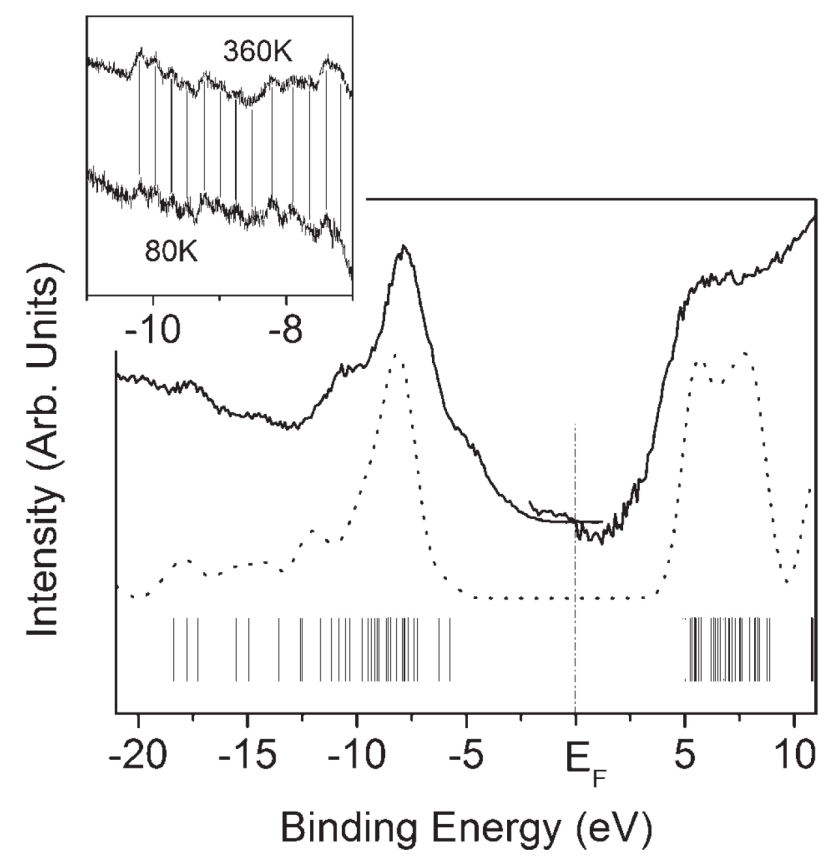

Figure 2. The combined UPS (left) and IPES (right) spectra, along with the calculated molecular orbitals (bottom lines) and model density of states (calculated using the configuration shown in Figure 1a) without any corrections for matrix element or cross-section effects (dashed middle line), are shown for PMVC (see text). The photoemission spectra were taken with the photoelectrons collected normal to the surface while the inverse photoemission spectrum was taken with the electrons at normal incidence. The inset shows a section of the spectra retaken with unpolarized light using a helium lamp at $h v=21.2 \mathrm{eV}(\mathrm{He} \mathrm{I})$. 


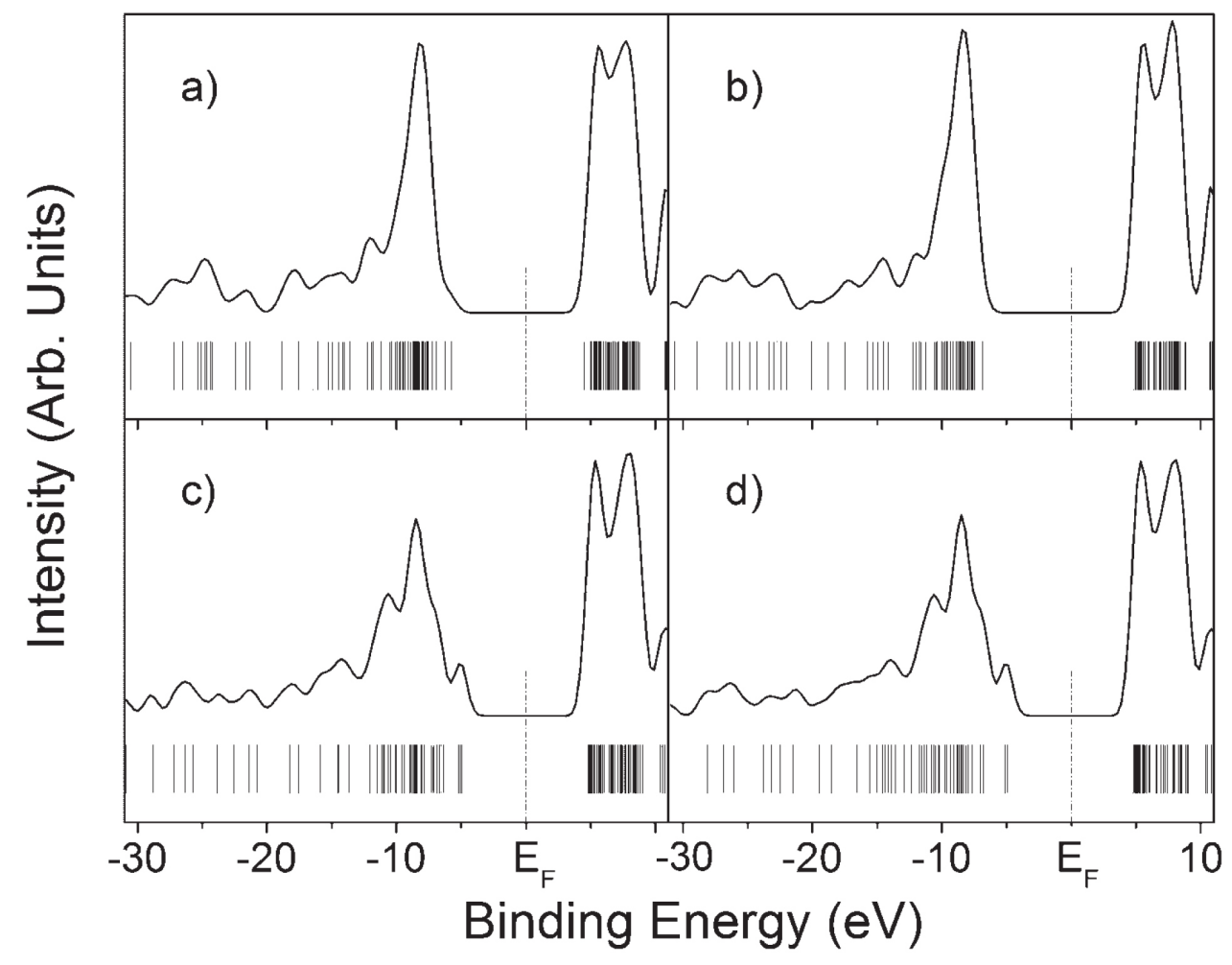

Figure 3. Semiempirical PM3 calculated molecular orbitals (bottom lines) and model density of states with $1 \mathrm{eV}$ Gaussian width added to the eigenvalues for four different configurations: (a) all-trans with methyl groups lined up; (b) all-trans with methyl groups on both sides alternatively; (c) alternating trans-gauche with methyl groups on both sides; and (d) alternating trans-gauche with methyl groups on one side; schematically shown for chain segments of PMVC in Figure 1.

\section{Comparison of Experiment and Theory}

While the Langmuir-Blodgett grown films of PMVC could adopt a geometric structure with all dipoles pointing to one direction and all the methyl groups sitting on one side of molecule, as shown schematically in Figure 1a, in reality, methyl groups need not be placed on one side: other possibilities exist such as a regio-regular alternating placement of methyl groups shown in Figure 1b. In both of these configuration examples, the carbon-carbon backbone of PMVC adopts the all-trans conformations, and dipole orientations are similar. Model calculations suggest that while the structural isomers are quite different, the molecular orbital DOSs are similar (Figures 3 and 4).

The alternating trans-gauche conformation is also possible for the polymer structure in ordered LangmuirBlodgett films of PMVC, just as is the case for P(VDFTrFE) in the paraelectric phase [3,4]. Similarly, the methyl groups could be placed on one side of molecule (Figure 1d) or alternatively as a regio-regular alternating placement of methyl groups (Figure 1c). These two different alternating trans-gauche conformation cases also produce the similar DOSs (panels d and c, respectively, in Figures 3 and 4). The key is that the alternating trans-gauche conformations all provide much different DOSs from that of the all-trans conformation isomers (panels a and b in Figures 3 and 4).

The antiparallel dipole orientation (alternating transgauche conformation) produces additional narrow band below the Fermi level, which is absent for parallel dipole orientation (all-trans conformation). Experimentally, we do not observe this narrow and intense DOSs at the valence band maximum in the UPS [7], as seen in Figure 2. Rather, the experimental data are in good agreement with the theoretical calculations of all-trans conformations, independent of model calculation methodology (panels a and b in Figures 3 and 4). This agreement between the experimental electronic structure and the calculated model DOSs suggests that Langmuir-Blodgett PMVC films adopt the alltrans conformation with all dipoles aligned. The position of methyl groups remains, however, somewhat ambiguous and cannot be ascertained from this comparison.

$A b$ initio calculation generates four spectra according to four different conformations which fall into two categories - all-trans (Figure $4 \mathrm{a}$ and $\mathrm{b}$ ) and alternating transgauche conformations (Figure $4 \mathrm{c}$ and $\mathrm{d}$ ). The $a b$ initio calculation does produce more features in the simulated spectra directly obtained from the DOSs than is the case from the semiempirical PM3 model calculations. This is because the 


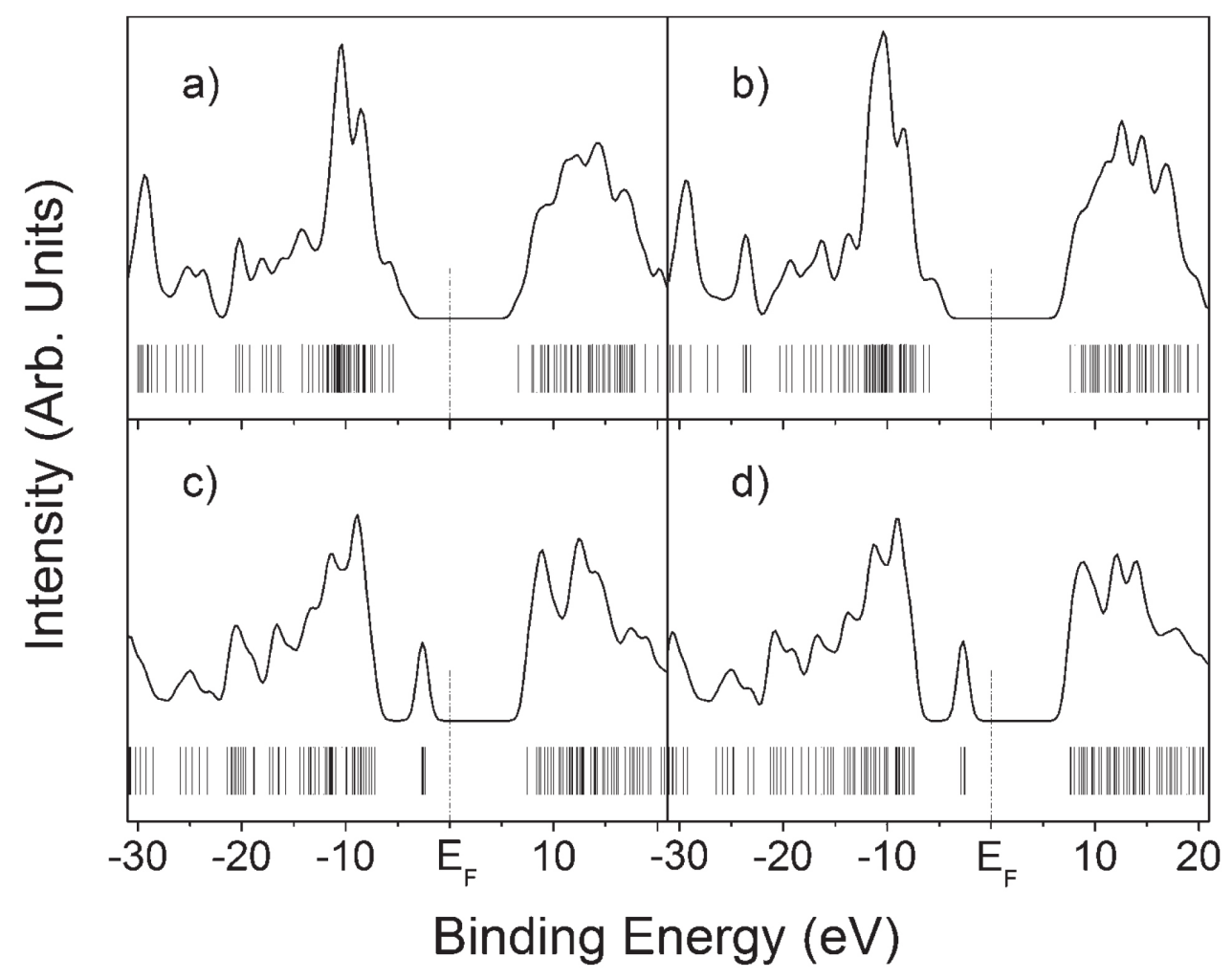

Figure 4. Ab initio method calculated molecular orbitals (bottom lines) and model density of states with $1 \mathrm{eV}$ Gaussian width added to the eigenvalues for four different configurations: (a) all-trans with methyl groups lined up; (b) all-trans with methyl groups on both sides alternatively; (c) alternating trans-gauche with methyl groups on both sides; and (d) alternating trans-gauche with methyl groups on one side; schematically shown for chain segments of PMVC in Figure 1.

inclusion of two-electron integrals in Hamiltonian splits some energy levels. These fine electronic structures were not observed in experiment, and in general, the semiempirical PM3 results fit experiment better-as is often the case [16], particularly with respect to the highest occupied to lowest unoccupied molecular orbital gap. In spite of the differences, both the $a b$ initio methods and the semiempirical PM3 calculations suggest that the all-trans conformation is the only structural phase of PMVC films at room temperature and below. Dipole ordering within a single chain that leads to a paraelectric phase seems unlikely as a result.

This view is supported by the energetics of the different configurations. The semiempirical PM3 and ab initio calculations also provide an estimate of the total energy of the four structural phases, which is the net result of electronic kinetic energies and the interactions between all electrons and atomic cores in the system. Both theoretical methodologies indicate that the total energies of two all-trans conformations are lower than that of two trans-gauche conformations, and hence favored. The total energies are similar for the two all-trans (or two trans-gauche) conformations, so we cannot conclusively distinguish between the various possible placements of the methyl groups in the all-trans configuration of the carbon-carbon backbone. Total energy calculations are done better using density functional the- ory, but even so, such calculations remain ambiguous - the films are grown on a graphite substrate and may be thus stabilized robustly in a metastable configuration, not necessarily the lowest energy configuration of a free polymer chain. Such interactions are not necessarily a result of strong chemical bonds, as even substrate image dipole interactions can play a role in the molecular configuration [28].

It is important to note that the vibrational fine structure seen in high resolution photoemission (Figure 2, inset) shows no temperature dependence, unlike the ferroelectric polymer thin films of the copolymer poly(vinylidene fluoride with trifluoroethylene) $\mathrm{P}(\mathrm{VDF}-\mathrm{TrFE})$ [2], where changing dipole orientation and dipole rigidity with respect to the film surface normal results in changing selection rules. This may be a result of the point group symmetry, but there is no indication whatsoever of any structure change in the vicinity of room temperature.

\section{Conclusion}

Both semiempirical PM3 and ab initio LCAO methods demonstrate that the dipole orientation can significantly alter the molecular orbital derived DOSs. The comparison of theory with experiment is sufficient to exclude the presence 
of a significant number of gauche bonds (i.e., such bonds must be below 5\%). The electronic structures of PMVC are indicative of systems in the all-trans configuration, with the dipoles all aligned. Structural phases that look like "paraelectric" and "antiferroelectric" are considered very unlikely, though antiparallel packing of PMVC chains cannot be excluded on the basis of a comparison of photoemission and inverse photoemission with theory. All-trans conformations are also seen to be energetically more stable than trans-gauche conformations thermodynamically, and are also, therefore, the more preferred configuration for ordered PMVC thin films, but the influence of the substrate in establishing thermodynamic stability needs to be explored.

\section{Acknowledgments}

The authors are grateful to S. Ducharme for his technical assistance and contributions. The LB films were made by M. Poulsen and S. Ducharme.

\section{References}

1. J. Choi, S.-J. Tang, P. T. Sprunger, P. A. Dowben, V. M. Fridkin, A. V. Sorokin, S. P. Palto, N. Petukhova, and S. G. Yudin, J. Phys. : Condens. Matter, 12, 4735 (2000).

2. L. G. Rosa, Ya. B. Losovyj, J. Choi, and P. A. Dowben, J. Phys. Chem. B, 109, 7817 (2005).

3. (a) L. M. Blinov, V. M. Fridkin, S. P. Palto, A. V. Bune, P. A. Dowben, and S. Ducharme, Usp. Fiz. Nauk (Russian edition volume), 170, 247 (2000); (b) L. M. Blinov, V. M. Fridkin, S. P. Palto, A. V. Bune, P. A. Dowben, and S. Ducharme, Phys. Usp. (English edition volume), 43, 243 (2000).

4. S. Ducharme, S. P. Palto, and V. M. Fridkin, "Ferroelectric and Dielectric Films," in Handbook of Surfaces and Interfaces of Materials, Vol. 3, H. S. Nalwa, Ed., Academic Press, New York, 546 (2002).

5. A. V. Bune, V. M. Fridkin, S. Ducharme, L. M. Blinov, S. P. Palto, A. V. Sorokin, S. G. Yudin, and A. Zlatkin, Nature, 391, 874 (1998).

6. C. N. Borca, T. Komesu, and P. A. Dowben, J. Electron Spectrosc. Relat. Phenom., 122, 259 (2002).

7. J. Xiao, L. G. Rosa, M. Poulsen, D. Q. Feng, D. S. Reddy, J. M. Takacs, L. Cai, J. Zhang, S. Ducharme, and P. A. Dowben, J. Phys. Condens. Matter., 18, L155 (2006).

8. J. Choi, P. A. Dowben, S. Ducharme, V. M. Fridkin, S. P. Palto, N. Petukhova, and S. G. Yudin, Phys. Lett. A, 249, 505 (1998).
9. J. Choi, C. N. Borca, P. A. Dowben, A. Bune, M. Poulsen, S. Pebley, S. Adenwalla, S. Ducharme, L. Robertson, V. M. Fridkin, S. P. Palto, N. N. Petukhova, and S. G. Yudin, Phys. Rev. B, 61, 5760 (2000).

10. M. Poulsen, S. Ducharme, A. V. Sorokin, S. Reddy, J. M. Takacs, Y. Wen, J. Kim, and S. Adenwalla, Ferroelectrics Lett., 32, 91 (2005).

11. J. Hormes, J. D. Scott, and V. P. Suller, Synchrotron Radiat. News, 19, 27 (2006).

12. Y. Losovyj, I. Ketsman, E. Morikawa, Z. Wang, J. Tang, and P. Dowben, Nucl. Instrum. Methods Phys. Res. A, 582, 264 (2007).

13. P. A. Dowben, D. LaGraffe, and M. Onellion, J. Phys. Condens. Matter, 1, 6571 (1989).

14. Y. Losovyj, K. Morris, L. Rosa, J. D. Scott, and P. Dowben, Nucl. Instrum. Methods Phys. Res. A, 582, 258 (2007).

15. J. J. P. Stewart, "PM3," in Encyclopedia of Computational Chemistry, Wiley, Athens, USA (1998).

16. I. N. Yakovkin and P. A. Dowben, Surf. Rev. Lett., 14, 481 (2007).

17. J. Muscat, A. Wander, and N. M. Harrison, Chem. Phys. Lett., 342, 397 (2001).

18. W. F. Perger, Chem. Phys. Lett., 368, 319 (2003).

19. Victor M. Bermudez, Naval Research Laboratories, private communication.

20. L. Cai, X. Wang, Y. Darici, P. A. Dowben, and J. Zhang, J. Chem. Phys., 126, 124908 (2007).

21. S. Lee, D. Li, P. A. Dowben, F. Keith Perkins, M. Onellion, and J. T. Spencer, J. Am. Chem. Soc., 113, 8444 (1991).

22. C. W. Hutchings, A. P. Hitchcock, A. T. Wen, S.-D. Hwang, J. A. Glass, J. T. Spencer, Y.-F. Hu, G. M. Bancroft, and P. A. Dowben, J. Electron. Spectrosc. Relat. Phenom., 94, 187 (1998).

23. D. K. Chambers, S. Karanam, D. Qi, S. Selmic, Ya. B. Losovyj, L. G. Rosa, and P. A. Dowben, Appl. Phys. A, 80, 483 (2005).

24. D.-Q. Feng, A. N. Caruso, D. L. Schulz, Ya. B. Losovyj, and P. A. Dowben, J. Phys. Chem. B, 109, 16382 (2005).

25. D.-Q. Feng, D. Wisbey, Ya. B. Losovyj, Y. Tai, M. Zharnikov, and P. A. Dowben, Phys. Rev. B, 74, 165425 (2006).

26. D. Wisbey, D. Feng, M. T. Bremer, C. N. Borca, A. N. Caruso, C. M. Silvernail, J. Belot, E. Vescovo, L. Ranno, and P. A. Dowben, J. Am. Chem Soc., 129, 6249 (2007).

27. D.-Q. Feng, A. N. Caruso, Ya. B. Losovyj, D. L. Schulz, and P. A. Dowben, Polym. Eng. Sci., 47, 1359 (2007).

28. C.-G. Duan, W. N. Mei, W.-G. Yin, J. Liu, J. R. Hardy, S. Ducharme, and P. A. Dowben, Phys. Rev. B, 69, 235106 (2004). 